\title{
Piperine Improves Obesity By Repairing Intestinal Barrier Function And Inhibiting Fatty Acid Absorption
}

\section{Wenli Wang}

China Agricultural University

\section{Yanhua Zhang}

China Agricultural University

\section{Xiong Wang}

China Agricultural University

Huilian Che

China Agricultural University

Yali Zhang ( $\square$ zhangyali@cau.edu.cn )

China Agricultural University

\section{Research}

Keywords: piperine, obesity, intestinal barrier, fatty acid absorption

Posted Date: April 27th, 2021

DOI: https://doi.org/10.21203/rs.3.rs-416340/v1

License: (c) (1) This work is licensed under a Creative Commons Attribution 4.0 International License. Read Full License

Version of Record: A version of this preprint was published at Plant Foods for Human Nutrition on September 30th, 2021. See the published version at https://doi.org/10.1007/s11130-021-00919-2. 


\section{Abstract}

\section{Background}

Currently, the weight loss effects of piperine have gained considerable attention; however, the underlying mechanism needs to be comprehensively elucidated. In the present study, we aimed to investigate the relationship between the weight loss effects of piperine and intestinal function.

\section{Methods}

Eight-week-old Sprague Dawley male rats were provided standard diet or HFD diet for 16 weeks. After, rats from the HFD group were divided into four group, including HFD, HFD with daily gavage with $2.7 \mathrm{mg} / \mathrm{kg}$ body weight of piperine (PIP-L), $13.5 \mathrm{mg} / \mathrm{kg}$ body weight of piperine (PIP-M), $27 \mathrm{mg} / \mathrm{kg}$ body weight of piperine (PIP-H) for another 8 weeks. The fecal fat content, serum TG, FAA levels, jejunum structure and gene expression levels related to fatty acid absorption and barrier function in intestinal were detected. Then the Caco-2 cell was cultured to explore the effects of piperine on cell proliferation, differentiation, barrier function and fatty acid absorption.

\section{Results}

In our study, piperine repaired the tight junction damage induced by obesity by downregulating jejunal tumor necrosis factor-a and reducing lipopolysaccharide-induced damage on intestinal cell proliferation, thus enhancing intestinal barrier function, which is beneficial in reducing chronic inflammation associated with obesity. In addition, piperine inhibited intestinal fatty acid absorption in both cellular and animal models. The underlying mechanism may be related to the downregulation of fatty acid absorption-related genes, fatty acid-binding protein 2 and cluster of differentiation 36 , but not fatty acid transport protein 4.

\section{Conclusion}

The anti-obesity effect of piperine is related to the enhancement of intestinal barrier function and inhibition of intestinal fatty acid absorption.

\section{Introduction}

According to recent global estimates by the World Health Organization, the worldwide prevalence of obesity has nearly tripled between 1975 and 2016. Once considered a high-income country problem, overweight and obesity are now on the rise in low- and middle-income countries, particularly in urban settings,and obesity has been a major risk factor for non-communicable diseases, including cardiovascular diseases, diabetes, musculoskeletal disorders, and some cancers.

Furthermore, obesity is associated with disorders of intestinal function, including barrier and absorption functions. The intestinal tract is the main area for digestion and absorption of nutrients, and the 
absorptive intestinal epithelium also functions as a tight barrier that limits the entry of pathogens and related toxins[1]. Diet-induced obesity is associated with increased intestinal permeability, which may be induced by altered gut microbiota[2]. The gut microflora and the consequently increased bacteria-derived factor, lipopolysaccharide (LPS), increase intestinal permeability by reducing the expression of epithelial tight junctions [3]through the toll-like receptor 4 (TLR4) signaling pathway[4]. Furthermore, in mice models of obesity and diabetes, hyperglycemia reportedly promotes intestinal barrier permeability through glucose transporter 2 (GLUT2)-dependent transcriptional reprogramming of intestinal epithelial cells and alters the integrity of tight and adhesive junctions[5]. In obesity, systemic low-grade inflammation is also closely associated with damage to the intestinal barrier function[5].

Obesity is the result of excessive fat accumulation, and the intestinal tract is the key location for fat absorption; thus, reducing the intestinal absorption of fatty acids is an important strategy to improve obesity. Previous research has reported that the small intestines adapt to the increased availability of dietary lipids, with the capacity for lipid digestion and absorption increasing accordingly[6]. Furthermore, studies have found that high-fat diet-induced jejunal microbiota directly increased gut lipid absorption in germ-free mice, independent of the diet consumed by experimental mice [7]. These results indicate that patients with obesity induced by a high-fat diet may possess a robust fatty acid absorption capacity than lean individuals, which can exacerbate obesity and related diseases. With a deepening understanding of obesity, novel therapeutic strategies are warranted. In addition to improving the composition of the gut microflora, regulating the fatty acid absorption capacity of intestinal epithelial cells is an important approach to lose weight. The mechanism and related signaling pathways of intestinal fatty acid absorption have been widely studied, and help provide a new target for the treatment of obesity by inhibiting intestinal fatty acid absorption. Stremmel et al. [8] have designed a new phospholipase inhibitor that could suppress the activation of $\mathrm{p}-\mathrm{JNK} 1$, a gene that regulates intestinal fatty acid. This inhibitor could be employed to treat obesity. Recently, increasing attention has been focused on the application of natural components from food and/or herbal medicines that are safer than chemical compounds in obesity management.

Piperine (PIP) is the first and most common amide alkaloid found to occur in pepper. The molecular formula is $\mathrm{C} 17 \mathrm{H} 19 \mathrm{NO} 3$ (Fig. 1). PIP has been found to possess antioxidant, immunomodulatory, antitumor, and drug metabolism effects ${ }^{9}$. Several studies have shown that PIP has a certain therapeutic effect on obesity ${ }^{9}$. Reportedly, supplemented PIP could improve changes in body weight, body composition, fat percentage, fat index, leptin, and adiponectin in obese rats induced by employing a highfat diet[11]. Current studies have observed that PIP can reduce energy intake by affecting the expression of appetite-suppressing genes such as transient receptor potential ion channels-vanillic acid receptor subtype 1 (TRPV1) and 5-hydroxytryptamine (5-HT)[11]. Furthermore, PIP can regulate genes related to lipid metabolism, including the downregulation of peroxisome proliferator-activated receptor $(P P A R)-\gamma$, acetyl CoA carboxylase $(A C C)$, fatty acid synthase $(F A S N)$, and Niemann-Pick C1-like 1 protein (NPC1L1) $[13,14]$. In addition to improving obesity by regulating lipid metabolism, piperine may also play a role by affecting intestinal function. ZhaoYimin et al [15]found that piperine can reduce intestinal cholesterol 
absorption by inhibiting the expression of intestinal NPC1L1, acyl-CoA cholesterol acyltransferase 2 (ACAT2) and microsomal triacylglycerol transport protein (MTP). Besides, the anti-inflammatory effect of piperine may have a protective effect on the damage of intestinal barrier. However, the mechanism of PIP underlying improvements in obesity needs to be further investigated.

The objective of the present study was to investigate the effects of PIP on intestinal barrier function and fatty acid absorption in obese rats and human colon adenocarcinoma cell lines (Caco-2). We presented evidence confirming that PIP improves obesity by repairing intestinal barrier damage and suppressing intestinal fatty acid absorption.

\section{Methods And Material}

\subsection{Animal and treatment}

Eight-week-old male Sprague Dawley rats (Beijing vital river Laboratory Animal Technology Co., Ltd.) were housed with two rats per cage, in a controlled environment (12:12-h light: dark cycle; temperature: $22^{\circ} \mathrm{C} \pm$ $2^{\circ} \mathrm{C}$; humidity: $60 \%$; $15-20$ fresh air changes per hour) with free access to food and water. After one week acclimatization period, 15 mice were randomly selected and fed with a standard diet assigned as the Chow group, whereas the remainder rats were placed on a high-fat and high-sugar diet for 16 weeks to induce obesity. The standard diet provided $3.42 \mathrm{kcal} / \mathrm{g}$ of energy $(19.2 \%$ from protein, $4.6 \% \mathrm{from}$ fat, $55.9 \%$ from carbohydrate), whereas the high-fat and high-sugar diet provided $4.62 \mathrm{kcal} / \mathrm{g}$ of energy (13.4\% from protein, $18.2 \%$ from fat, $54.1 \%$ from carbohydrate). After a 16 -week feeding period on the high-fat and high-sugar diet, 40 rats with the highest weight were randomly reassigned to four groups (n $=10$, respectively) and either fed a high-fat and high-sugar diet (HFHS group) or the high-fat and highsugar diet with oral administration of low-dose PIP ( $2.7 \mathrm{mg} / \mathrm{kg}$ body weight, PIP-L), middle-dose PIP (13.5 $\mathrm{mg} / \mathrm{kg}$ body weight, PIP-M), high-dose PIP (27 mg/kg body weight, PIP-H), respectively, from day 1 for another 8 weeks. PIP (purity $\geq 99 \%$, Sigma, America) was suspended in $0.5 \%$ sodium carboxymethyl cellulose (CMC-Na), whereas the same amount of $0.5 \%$ CMC-Na was orally administered to the HFHS and Chow groups (Table 1). It has been reported that the average consumption of black pepper is approximately $0.7 \mathrm{~g} /$ person/day in the USA, indicating that the daily consumption of PIP is nearly 14-54 $\mathrm{mg} /$ person[16]. In this experiment, we converted the daily per capita consumption of black pepper into the equivalent rat dose[17] as the low-dose of PIP $(2.7 \mathrm{mg} / \mathrm{mg} / \mathrm{kg})$. Simultaneously, 5 -fold the estimated rat dose $(13.5 \mathrm{mg} / \mathrm{kg})$ was deemed the medium-dose, with 10 -fold $(27 \mathrm{mg} / \mathrm{kg})$ as high-dose, to observe the effects of different PIP doses on obese rats. All the rats were treated in accordance with the guidelines of the Institutional Animal Care and Use Committee, and all experiments were approved by the Animal Experimentation Committee of the China Agricultural University (Beijing, China).

\subsection{Sample collection}

At the end of the experiment, a fresh fecal sample was collected, freeze-dried into a powder, and stored in a refrigerator at $-80^{\circ} \mathrm{C}$. Rats were sacrificed under anesthesia with isoflurane without fasting, and the 
jejunum was collected for sectioning and real-time polymerase chain reaction (RT-PCR), serum was separated by centrifugation $\left(4^{\circ} \mathrm{C}, 3000 \mathrm{~g}, 15 \mathrm{~min}\right)$ and stored at $-80^{\circ} \mathrm{C}$.

\subsection{Determination of fecal fat content}

After accurately weighing $0.1 \mathrm{~g}$ of a fecal sample, $0.4 \mathrm{~mL}$ distilled water, $1 \mathrm{~mL}$ distilled water, and $0.6 \mathrm{~mL}$ concentrated hydrochloric acid was added, mixing well at each step. After heating at $80^{\circ} \mathrm{C}$ for 50 min in a water bath, $3 \mathrm{~mL}$ ether was added to the sample, and then plugged and inverted for 2-3 min repeatedly (approximately 60-80 times) after cooling to ensure thorough mixing. After standing for $15 \mathrm{~min}$, the supernatant was absorbed in a constant-weight evaporation dish and dried in an oven at $105^{\circ} \mathrm{C}$ to a constant weight. The weight was accurately weighed on an analytical balance. The following formula was used to determine the fat content in the feces. Fecal fat content $=$ (evaporating dish + fat constant weight - evaporating dish constant weight)/fecal weight $\times 100 \%$.

\subsection{RT-PCR analysis}

RT-PCR analysis was performed as previously described. Total RNA was extracted from tissue using a HiPure Total RNA Mini Kit (Magen, Guangzhou, China) according to the manufacturer's instructions. The concentration of RNA was detected by measuring the A260/A280 and A260/A230 ratios with Nanodrop2000 (Thermo Scientific, Thermo Fisher Scientific, Waltham, USA). Total RNA (1 $\mu \mathrm{g}, 10 \mu \mathrm{L})$ was reverse transcribed using a HiFi Script gDNA Removal cDNA Synthesis Kit (CWBIO, Beijing, China). The RT-PCR reaction was performed using Rotor-Gene Q (Qiagen, Hilden, Germany) with an Ultra SYBR Mixture Kit (CWBIO, Beijing, China). RT-PCR amplification conditions included denaturation at $95^{\circ} \mathrm{C}$ for 10 $\mathrm{s}$, annealing at $58^{\circ} \mathrm{C}$ for $30 \mathrm{~s}$, and extension for $32 \mathrm{~s}$ at $72^{\circ} \mathrm{C}$. Expression of Cluster differentiation $36(C D-$ 36), fatty acid transport protein 4 (FATP4), fatty acid-binding protein 2 , also called intestinal fatty acidbinding protein ( $F A B P 2, I-F A B P)$, tumor necrosis factor-alpha (TNF-a), and two tight junction proteins, including zonula occludens $(Z O)-1$ and occluding, were quantified using GAPDH as the housekeeping gene[18]. Sequences of primers used in this study are listed in Table 2.

Table 2

List of Primer Sequences

\begin{tabular}{|lll|}
\hline Gene & Forward & Reverse \\
\hline CD-36 & GGTCCTTACACATACAGAGT & CCACAGCCAGATTGAGAA \\
\hline FATP4 & GGCACTCATCAACACCAA & CAGGAACCAGAGCAGAAG \\
\hline FABP2 & AATGTGGTGAAGAGGAAGC & TGTGTAGGTCTGGATTAGTTC \\
\hline ZO-1 & ACAGCTATATGGGAACAGCAC & ACTATGGAGGTTTCCCCACTC \\
\hline Occludin & TTGAGAGTCCACCTCCTTA & CTGATGAGAGGGAGCCCATTTG \\
\hline TNF-a & CCACCACGCTCTTCTGTCTAC & CTGATGAGAGGGAGCCCATTTG \\
\hline GAPDH & CTCTCTGCTCCTCCCTGTTCTA & CCGACCTTCACCATCTTGTCTA \\
\hline
\end{tabular}




\subsection{Cell culture}

The Caco-2 cell line was procured from ATCC and used between the 20th and 50th passages. Cells were grown in Dulbecco's modified Eagle's medium (DMEM; Corning, NY, USA) with a high glucose concentration $(4.5 \mathrm{~g} / \mathrm{L})$, and supplemented with $10 \%$ fetal bovine serum (FBS, VISTECH, New Zealand), $1 \%$ nonessential amino acids (Gibco, USA), and $1 \%$ penicillin-streptomycin mixture (Gibco, USA), at $37^{\circ} \mathrm{C}$ under a $5 \% \mathrm{CO}_{2}-95 \%$ air atmosphere in $25 \mathrm{~cm}^{2}$ plastic tissue culture flasks (Corning, NY, USA). For Caco-2 cells, the medium used was complete culture medium, which was replaced every 2-3 days. For subculture, the confluent cells were isolated by treatment with trypsin with $0.25 \%$ EDTA (Corning, NY, USA), diluted in complete culture medium.

\subsection{Cell proliferation measurements}

Caco-2 cells were seeded in 96 plates at an initial cell density of $1.5 \times 10^{4}$ cells/well and cultured for $24 \mathrm{~h}$ in complete culture medium. Cells were exposed to increasing doses of PIP (mother liquid soluble in dimethyl sulfoxide) dissolved in complete culture medium for $24 \mathrm{~h}$. Cells in complete culture medium without PIP were used as the control group. The cell proliferation assay was performed using the CCK8 Assay kit (Beyotime Biotechnology, Jiangsu, China). Cell proliferation was calculated by relative absorbance compared to the untreated group using the following formula: Cell viability $(\%)=$ Average (absorbance with PIP treatment - blank)/Average (absorbance without PIP treatment - blank) $\times 100 \%$. The assay was performed in triplicate.

\subsection{Suppression of cell proliferation by LPS}

Caco-2 cells were seeded in 96 plates at an initial cell density of $1.5 \times 10^{4}$ cells/well and cultured for $24 \mathrm{~h}$ in complete culture medium. Cells were exposed to PIP for $24 \mathrm{~h}$ Subsequently, $400 \mu \mathrm{g} / \mathrm{ml}$ LPS was added for $24 \mathrm{~h}$, and then the cells were evaluated with the CCK8 assay. The method of operation was similar to that of PIP, and LPS was dissolved in complete medium.

\subsection{Cell differentiation measurements}

The alkaline phosphatase (ALP) activity was used as an index to determine the degree of cell differentiation. Caco-2 cells were seeded on 96 wells at an initial cell density of $1.5 \times 10^{4}$ cells/well and cultured in complete culture medium. When the Caco- 2 cells reached $90 \%$ confluence, complete medium was replaced by PIP dissolved in complete medium. Cells in complete medium without PIP were used as the control group. On day 14, ALP activity was measured using an alkaline phosphatase kit (Beyotime Biotechnology, Jiangsu, China).

\subsection{Establishment of monolayer barrier model of Caco-2 cells}

Caco-2 cells were seeded on transwell polycarbonate insert filters $(0.4 \mu \mathrm{m}$ pore size, $6.5 \mathrm{~mm}$ diameter; Corning Costar Corporation, Cambridge, MA) in 24-well plates at a density of $2.5 \times 10^{4}$ cells/well. Cells 
were allowed to grow for 21 days. For the first seven days, the medium was replaced every two days, and then, daily. The transepithelial electrical resistance (TEER) of monolayer cells was measured using Millicell ERS-2 (Millipore, MA, USA) according to the manufacturer's instructions, and TEER exceeding 250 $\Omega / \mathrm{cm}^{2}$ was used for the next experiment. According to the design of the control and the experimental groups, $100 \mu \mathrm{L}$ medium was added to the upper chamber and $600 \mu \mathrm{L}$ complete medium was added to the lower chamber. After several hours of culture, the resistance of each group was detected. TEER (\% from initial value $)=$ TEER (after treatment) $/$ TEER (before treatment) $\times 100 \%$. The in vitro intestinal permeability was measured using 4000 Da FITC-dextran (FD4000, Sigma-Aldrich, Missouri, USA), with $100 \mu \mathrm{L}$ FITC-dextran $(4000 \mathrm{Da}, 1 \mathrm{mg} / \mathrm{mL}$ ) was added into the upper chambers of the transwell and allowed to incubate for $1 \mathrm{~h}$ at $37^{\circ} \mathrm{C}$. The FITC concentration in the lower chamber was quantified and calculated against a standard curve (excitation $435 \mathrm{~nm}$, emission $490 \mathrm{~nm}$ ). $P_{\text {FITC-dextran } 4 \mathrm{kD}}=d Q / d t / A C$ ( $d Q=$ quality of FITC-dextran in the lower chamber, $d t=$ incubation time, $A=$ transwell growth area, $C=$ initial concentration in the upper chambers).

\subsection{In vitro determination of fatty acid uptake [19]}

Caco-2 cells were seeded in 96-well plates (black wells, clear bottom with low evaporation lid, Corning, NY, USA) for 1 week for the differentiation process. Fatty acid uptake was analyzed using the Fatty Acid Uptake Kit (Sigma-Aldrich, USA) as described previously [20]. The plates were maintained in the dark and incubated for $60 \mathrm{~min}$ at $37^{\circ} \mathrm{C}$ in $5 \% \mathrm{CO}_{2}$. Intracellular fluorescence was measured using a fluorescence microplate reader (Thermo Fisher Scientific, Waltham, MA, USA), with excitation at $485 \mathrm{~nm}$ and emission at $528 \mathrm{~nm}$. Differences in fatty acid uptake were compared by relative fluorescence.

\subsection{Statistical analysis}

Data are presented as mean \pm standard error of the mean (SEM). Statistical significance was determined by employing one-way ANOVA followed by Tukey's multiple-comparison test, using SPSS version 23.0 (IBM Corp., Armonk, NY, USA). All statistical data with $p<0.05$ were considered significant.

\section{Results}

\subsection{Effect of PIP on fat digestion and absorption in HFHS rats}

To determine whether PIP can inhibit intestinal digestion and absorption of fatty acids, we measured the fecal fat content of rats in the HFHS and PIP groups. As shown in Fig. 2A, the fecal fat content of each PIP group was significantly higher $(p<0.05)$ than that of the HFHS group. Compared with the Chow group, the serum levels of TG and FAA were significantly increased in the HFHS group. Compared with the HFHS group, the serum levels of TG and FAA were significantly decreased in PIP-M and PIP-H groups $(p<$ 0.01). Treatment with low concentration of piperine significantly reduced the level of FAA $(p<0.01)$, but 
had no effect on TG (Table 3). To further understand the underlying mechanism, the rat jejunum was collected and the transcriptional expression of several genes related to fatty acid absorption was measured. $C D-36, F A B P 2$, and FATP4 are wildly expressed in the small intestine and play an important role in fatty acid absorption. The data showed that PIP-H significantly decreased $(p<0.05) C D-36$ gene expression (Fig. 2B), which was 70\% lower than that of the HFHS group, and the PIP-L and PIP-M did not differ from HFHS. In the case of $F A B P 2$ expression in the rat jejunum, all three PIP doses revealed significantly lower expression $(p<0.05)$ than that observed in the HFHS group (Fig. 2C), decreasing by $68 \%, 57 \%$, and $52 \%$, respectively. Simultaneously, FATP4 expression was reduced, with no significant difference observed between the PIP and HFHS groups (Fig. 2D). Hematoxylin-eosin (H\&E)-stained sections from the jejunum revealed that the HFHS diet can significantly decrease the ratio of villus height to crypt depth $(\mathrm{VCR})(\mathrm{p}<0.05)$, but no significant difference was observed between the three PIP dose groups and the HFHS group (Fig. 2E).

Table 3

Effects of piperine on serum TG, FAA levels.

\begin{tabular}{|c|c|c|}
\hline & TG (mmol/L) & $\mathrm{FAA}(\mathrm{mmol} / \mathrm{L})$ \\
\hline Chow & $0.76 \pm 0.1$ & $0.37 \pm 0.03$ \\
\hline HFHS & $1.92 \pm 0.08^{* *}$ & $0.65 \pm 0.02^{* *}$ \\
\hline PIP-L & $2.02 \pm 0.12^{\star \star}$ & $0.61 \pm 0.02 b^{\star \star \# \# ~}$ \\
\hline PIP-M & $1.79 \pm 0.04^{\star \star \# \#}$ & $0.58 \pm 0.04^{* \star \# \#}$ \\
\hline PIP-H & $1.55 \pm 0.35^{\star \star \# \# ~}$ & $0.48 \pm 0.05^{\star \star \# \#}$ \\
\hline \multicolumn{3}{|c|}{$\begin{array}{l}\text { Data expressed as mean } \pm \text { SD }(n=9-10) .{ }^{*} p<0.05 \text { and } * * p<0.01 \text { versus Chow group; } \# p<0.05 \text { and } \\
\# \# p<0.01 \text { versus HFD group. }\end{array}$} \\
\hline
\end{tabular}

\subsection{Effect of PIP on intestinal barrier function}

Obesity is often associated with intestinal barrier damage[21]. We explored whether PIP improves intestinal barrier function while improving obesity. We detected the expression levels of tight junction markers $20-1$ and occludin, which play a primary role in maintaining intestinal barrier function (Fig. $2 \mathrm{~F}$, Fig. $2 \mathrm{G})$. The HFHS diet significantly decreased $(p<0.05)$ the expression of occludin when compared with that in the Chow group; PIP significantly increased $(p<0.05)$ the expression of occludin when compared with that in the HFHS group, and did not differ from that of the Chow group. However, no significant differences in ZO-1 expression were observed between the Chow, HFHS, and PIP groups. To further explore whether the effects of PIP on tight junction protein is related to its anti-inflammatory activity, jejunal expression of TNF- $a$ was determined. As shown in Fig. $1 \mathrm{H}$, the expression of TNF- $a$ in the jejunum of rats treated with the HFHS diet was significantly higher $(p<0.05)$ than that of rats in the Chow group, and PIP significantly downregulated $(p<0.05)$ the expression of TNF-a when compared with the HFHS group. 


\subsection{Effect of PIP on the proliferation of Caco-2 cells}

To explore the effects of PIP on Caco-2 cell proliferation, the cells were treated with PIP for $24 \mathrm{~h}$, and subsequently, viability was examined using the CCK-8 assay. PIP treatment, ranging from $0.1 \mu \mathrm{M}$ to 10 $\mu \mathrm{M}$, did not affect cell proliferation, inhibiting cell proliferation at a concentration of $100 \mu \mathrm{M}(p<0.05)$ (Fig. 3A).

Caco-2 cells were preincubated with PIP to explore the protective effect of PIP on LPS-induced damage. The experimental results showed that cell activity in the model group was significantly lower $(p<0.05)$ than that of the control group, indicating that the LPS damage model was successfully established. On adding $0.1 \mu \mathrm{M}, 10 \mu \mathrm{M}$, and $100 \mu \mathrm{M}$ PIP separately, the viability demonstrated no significant effect when compared with the model group; however, with $1 \mu \mathrm{M}$ PIP, viability was significantly higher $(p<0.05)$ than that of the model (Fig. 3B). The results showed that a specific PIP concentration presented a protective effect on LPS-induced cell activity damage.

\subsection{Effect of PIP on the differentiation of Caco-2 cells}

Alkaline phosphatase (ALP) is one of the polarization marker enzymes of Caco-2 cells, which is mainly expressed in the brush border of Caco-2 cells and can be used to monitor the degree of cell differentiation. At low concentrations, no difference was observed between the PIP and control groups. However, the ALP activity of the $10 \mu \mathrm{M}$ and $100 \mu \mathrm{M}$ PIP groups was significantly lower $(p<0.05)$ than that of the control group (Fig. $3 \mathrm{C}$ ). The results revealed that prolonged PIP treatment of cells might inhibit cell differentiation, suggesting that the dosage and time of PIP treatment should be strictly controlled.

\subsection{Effect of PIP on the barrier function of Caco-2 cell monolayers}

The barrier function of Caco-2 cell monolayers was detected by TEER and the permeability of FITCdextran through the monolayers. The Caco-2 cell monolayer was treated with $10 \mu \mathrm{M}$ PIP for $24 \mathrm{~h}$, and TEER increased by $25 \%$ when compared with the control group (Fig. 3D). However, PIP treatment did not affect the monolayer permeability (Fig. 3E).

\subsection{Effect of PIP on monolayer fatty acid absorption of Caco-2 cells}

To quantify the effect of PIP on cellular free fatty acid (FFA) uptake, the level of TF2-C12 fatty acids was measured. As indicated in Fig. 3F, $100 \mu \mathrm{M}$ PIP significantly reduced $(p<0.05)$ the fatty acid absorption by Caco-2 cell monolayers, when compared with the control group.

\section{Discussion}

An in-depth study on the biological activity of natural active substances provides a novel strategy for the treatment of several diseases. As an active substance derived from pepper and an extensively used spice, 
the anti-obesity effect of PIP has been widely reported [11, 14, 22]. However, the mechanism underlying the weight loss effect of PIP remains unclear, and there is still a long road ahead of clinical application. Based on previous research in our laboratory, PIP can significantly reduce body weight, as well as triglyceride (TG) and FFA levels in the blood of HFHS diet rats in a dose-dependent manner (unpublished results). In this study, we explored the effect of PIP on the jejunum of HFHS diet rats and Caco-2 cells in vitro from the perspective of intestinal function.

By employing a suitable rat model, our study revealed that PIP significantly increased the content of crude fat in feces under the same dietary conditions; thus, we speculate that PIP may inhibit the digestion and absorption of fat in the intestinal tract. In vitro cell experiments demonstrated that PIP suppressed fatty acid absorption by Caco-2 cells, confirming previous evidence. The structure of the rat jejunum was examined in our experiment, and no difference was observed in the ratio of villus height to crypt depth between the HFHS and PIP groups; however, PIP reduced the expression of genes related to fatty acid absorption in the jejunum, including $C D 36$ and $F A B P 2$. Collectively, the results indicated that the inhibitory effect of PIP on fatty acid absorption is specific. $C D 36$ is a transmembrane protein that is highly expressed on the apical membrane of villi enterocytes in the jejunum[23] and plays an important role in fatty acid uptake and chylomicron formation in enterocytes[23, 24]. Related research has demonstrated that intestinal very-long-chain fatty acid (VLCFA) absorption is completely abolished in CD36-null mice fed a high-fat diet[25]; therefore, downregulation of CD36 induced by PIP-H may reduce the absorption of dietary fat (Fig. 4). Interestingly, CD36 also plays an unsuspected role in maintaining the integrity of the epithelial barrier, and the small intestines of $C D 36 \mathrm{KO}$ mice fed a chow diet reportedly show compromised barrier function[1]. CD36 expression in the HFHS group was slightly lower than that in the Chow group (Fig. 2B), which may be related to the damage of the intestinal barrier induced by a high-fat diet. The relationship between the regulation of fatty acid absorption and the barrier function of $C D 36$ remains elusive, and the regulation of $C D 36$ mediated by PIP needs further study. The $F A B P$ family includes plasma membrane-associated $F A B P s(F A B P p m)$ and cytoplasmic $F A B P s(F A B P C)$, and $F A B P 2$ is a type of cytoplasmic $F A B P$ that is found exclusively in the small intestine[26]. Early studies have shown that the intestinal $F A B P$ concentration in animals fed a high-fat diet is significantly higher than that in animals fed a low-fat diet[27]. However, in our study, no significant difference was observed in the FABP2 expression between the HFHS and Chow groups, which may be related to differences between the highfat diet and HFHS diet. In the present study, the decrease in FABP2 expression induced by PIP treatment is beneficial for reducing the absorption and transport of fatty acids in the intestine. Furthermore, the downregulation of $F A B P 2$ may also have a positive effect on intestinal permeability. A recent study has revealed that intestinal $F A B P 2$ knockdown improves intestinal permeability by increasing the expression of tight junction proteins[28]. FATP4 is the only FATP expressed in the small intestine [29], and a range of fat and/or cholesterol-supplemented diets has revealed no effect on FATP4 mRNA or protein expression in the intestine of wild-type mice[30]. Similar to our results, no difference in the FATP4 expression between the two groups was observed, and PIP treatment did not affect the expression of FATP4. Intestinal fatty acid absorption is regulated by multiple genes. According to the results of this experiment, the decrease in intestinal fatty acid absorption induced by PIP was mainly related to the regulation of 
FABP2, and no compensatory increase in other gene expressions was detected. Other studies have indicated that PIP may also play a role by affecting intestinal function. ZhaoYimin et al. have observed that PIP can reduce intestinal cholesterol absorption by inhibiting the expression of intestinal NPC1L1, acyl-CoA cholesterol acyltransferase 2 (ACAT2), and microsomal triacylglycerol transport protein (MTP) [31]. Nevertheless, research has shown that piperine enhances the secretion of bile acids and also causes inhibition of bile acid metabolism [31], which may promote the absorption of lipids.

Impaired intestinal barrier induced by obesity has attracted extensive attention, emphasizing the mechanism through which the increase in intestinal permeability is associated with reduced expression of epithelial tight junction proteins such as Z0-1, occludin, and claudin [33]. In contrast to existing research results[3], no changes in the expression of $Z O-1$ were observed in the present study, which may be attributed to differences in intestinal sites. Treatment with PIP significantly recovered the decrease in tight junction expression induced by HFHS, which may be related to its modulatory potential in immune inflammation[34]. The pro-inflammatory cytokine TNF-a has been shown to directly impair tight junction function in epithelial cell lines[35]. In addition, the tight junction protein occludin, which is strongly regulated by $T N F-a$, is considered a general indicator of tight junction integrity[36]. In our study, PIP downregulated the expression of TNF- $a$ and reduced the damage induced by TNF- $a$ to the tight junction protein occludin. Another study has reported that TNF- $a$ downregulated the expression of ZO-1 and altered the junctional localization by activating nuclear factor-kappa B (NF-KB)[37]. Additionally, the TNF$a$ induced increase in intestinal tight junction permeability was mediated by an increase in myosin lightchain kinase (MLCK) protein expression[38]. Combined with these mechanisms of tight junction regulation by TNF- $a$, the underlying PIP mechanism that restores the expression of tight junction proteins by reducing the overexpression of TNF- $a$ deserves further investigation. To further explore the protective mechanism of PIP on the intestinal barrier, the effects of PIP on the proliferation, differentiation, and monolayer integrity of Caco-2 cells were explored. PIP did not promote proliferation and differentiation of Caco-2 cells, and a high concentration of PIP showed cytotoxicity, inhibiting cell proliferation and differentiation. Although PIP treatment increased TEER of Caco-2 cell monolayers, no significant difference was observed in intestinal epithelial permeability in Caco-2 cells, which may be related to the low molecular weight of FITC-dextran selected in this experiment. However, following PIP pretreatment, cell proliferation was increased at certain concentrations when compared to those in the LPS treatment, indicating that PIP had an obvious protective effect on Caco2 cell damage induced by LPS. Based on our findings, we hypothesized that PIP has no effect on the intestinal barrier function of healthy individuals, but when the intestinal barrier is damaged, it may protect intestinal barrier integrity by reducing the decrease in cell activity induced by the injury. PIP has been reported to significantly ameliorate TNBSinduced colonic aberrations, inhibiting the overexpression of proinflammatory cytokines (TNF- $a$ and interleukins), which may improve the expression of tight junction proteins (Claudin-1, occludin, and ZO-1) [39]. In addition to the factors explored in this study, the antioxidant activity of piperine may also be an important reason for piperine to repair intestinal barrier. Early studies have shown that the intake of high doses of polyunsaturated fatty acids can promote lipid peroxidation and the subsequent propagation of 
oxygen radicals which is the main factor responsible for the loss of epithelial barrier function through tyrosine phosphorylation and redistribution of tight junction proteins [40].

\section{Conclusions}

In conclusion, this study demonstrated that PIP improves obesity by repairing intestinal barrier injury and suppressing intestinal fatty acid absorption. PIP improved the intestinal barrier function of obese rats by reducing LPS-induced damage to intestinal cell proliferation, downregulating the overexpression of proinflammatory cytokine TNF- $a$, and improving the decreased expression of tight junction proteins induced by obesity. Furthermore, PIP reduced the expression of fatty acid absorption-related proteins such as FABP2 and $C D 36$ by inhibiting the absorption of fatty acids in intestinal epithelial cells. PIP achieves weight loss through the restriction of fatty acid intake and transportation.

\section{Abbreviations}




\begin{tabular}{|c|c|}
\hline LPS & lipopolysaccharide \\
\hline TLR4 & Toll-like receptor 4 \\
\hline GLUT2 & glucose transporter 2 \\
\hline PIP & piperine \\
\hline TRPV1 & transient receptor potential ion channels-vanillic acid receptor subtype 1 \\
\hline $5-\mathrm{HT}$ & 5-hydroxytryptamine \\
\hline PPAR- $Y$ & Peroxisome proliferators activated receptors \\
\hline ACC & Acetyl CoA Carboxylase \\
\hline FASN & fatty acid synthase \\
\hline NPC1L1 & Niemann-Pick C1-like 1 protein \\
\hline Caco-2 & human colon adenocarcinoma cell lines \\
\hline HFHS & high-fat and high-sugar \\
\hline$C D-36$ & Cluster determinant 36 \\
\hline FATP4 & fatty acid transport protein 4 \\
\hline$F A B P 2 / I-F A B P$ & fatty acid-binding protein 2 /intestinal fatty acid-binding protein \\
\hline$T N F-a$ & tumor necrosis factor alpha \\
\hline $20-1$ & zonula occludens- 1 \\
\hline$A L P$ & alkaline phosphatase \\
\hline TEER & transepithelial electrical resistance \\
\hline$V C R$ & ratio of villus height to crypt depth \\
\hline$T J$ & Tight Junction \\
\hline$T G$ & triglycerides \\
\hline FFA & free fatty acids \\
\hline VLCFAs & very long chain FAs \\
\hline ACAT2 & acyl-CoA cholesterol acyltransferase 2 \\
\hline MTP & microsomal triacylglycerol transport protein \\
\hline MLCK & myosin light-chain kinase \\
\hline FABP pm & plasma membrane-associated $F A B P s$ \\
\hline$F A B P C$ & cytoplasmic FABPS \\
\hline
\end{tabular}




\begin{tabular}{|ll|} 
PCTV & prechylomicron transport vesicle \\
\hline ER & endoplasmic reticulum \\
\hline
\end{tabular}

\section{Declarations}

\section{Ethics approval and consent to participate}

In our research, no human experiments were involved. All animal experiments were performed according to the Animal Care and Use Committee (permission SCXK 2016- 0006) and the guidelines for Animal Experiments at China Agricultural University.

\section{Consent for publication}

Not applicable

\section{Availability of data materials}

The datasets used in the current study are available from the corresponding author on reasonable request.

\section{Competing interests}

The authors declare that they have no known competing financial interests or personal relationships that could have appeared to influence the work reported in this paper.

\section{Funding}

This work was supported by the Program of the National Natural Science Foundation of China (Grant No.81072305), the Major Program of the National Natural Science Foundation of China (Grant No.31790412).

\section{Author contributions}

Wenli Wang: Conceptualization, Data curation, Formal analysis, Writing-Original draft preparation, Writing-Reviewing and Editing. Yanhua Zhang: Investigation, Methodology, Data curation. Xiong Wang: Visualization, Investigation. Huilian Che: Reviewing. Yali Zhang: Funding acquisition, Project administration, Reviewing.

\section{Acknowledgment}

We would like to thank Editage (www.editage.cn) for English language editing.

\section{References}


1. Cifarelli V, Ivanov S, Xie Y, Son N, Saunders BT, Pietka TA, Shew TM, Yoshino J, Sundaresan S, Davidson NO, Goldberg IJ, Gelman AE, Zinselmeyer BH, Randolph GJ, Abumrad NA. CD36 Deficiency Impairs the Small Intestinal Barrier and Induces Subclinical Inflammation in Mice. Cellular Molecular Gastroenterology Hepatology. 2017;3:82-98.

2. Winer DA, Luck H, Tsai S, Winer S. The Intestinal Immune System in Obesity and Insulin Resistance. Cell Metaboliam. 2016;23:413-26.

3. Cani PD, Bibiloni R, Knauf C, Waget A, Neyrinck AM, Delzenne NM, Burcelin R. Changes in Gut Microbiota Control Metabolic Endotoxemia-Induced Inflammation in High-Fat Diet-Induced Obesity and Diabetes in Mice. Diabetes. 2008;57:1470-81.

4. Kim K, Lee WGI, Joh E, Kim D. High Fat Diet-Induced Gut Microbiota Exacerbates Inflammation and Obesity in Mice via the TLR4 Signaling Pathway. Plos One. 2012;7:1-11.

5. Thaiss CA, Levy M, Grosheva I, Zheng D, Soffer E, Blacher E, Braverman S, Tengeler AC, Barak O, Elazar M, Ben-Zeev R, Lehavi-Regev D, Katz MN, Pevsner-Fischer M, Gertler A, Halpern Z, Harmelin A, Aamar S, Serradas P, Grosfeld A, Shapiro H, Geiger B, Elinav E. Hyperglycemia drives intestinal barrier dysfunction and risk for enteric infection. Science. 2018;359:1376-83.

6. Singh A, Rodgers JB, Balint JA, Ednonds RH. Adaptive change of rat small-intestinal in response to a high fat diet. Biochim Biophys Acta. 1972;260:708-15.

7. Martinez-Guryn K, Hubert N, Frazier K, Urlass S, Musch MW, Ojeda P, Pierre JF, Miyoshi J, Sontag TJ, Cham CM, Reardon CA, Leone V, Chang EB. Small Intestine Microbiota Regulate Host Digestive and Absorptive Adaptive Responses to Dietary Lipids. Cell Host Microbe. 2018;23:458-69.

8. Stremmel W, Staffer S, Wannhoff A, Pathil A. The overall fatty acid absorption controlled by basolateral chylomicron excretion under regulation of p-JNK1. BBA - Molecular Cell Biology of Lipids. 2017; 1862:917-28.

9. Atal S, Agrawal RP, Vyas S, Phadnis P, Rai N. Evaluation of the Effect of Piperine Perse on Blood Glucose Level in Alloxan-linduced Diabetic Mice. Acta Pol Pharm. 2012;69:965-9.

10. Meriga B, Parim B, Chunduri VR, Naik RR, Nemani H, Suresh P, Ganapathy S, Uddandrao VVS. Antiobesity potential of Piperonal: promising modulation of body composition, lipid profiles and obesogenic marker expression in HFD-induced obese rats. Nutrition Metabolism. 2017;14:72.

11. BrahmaNaidu P, Nemani H, Meriga B, Mehar SK, Potana S, Ramgopalrao S. Mitigating efficacy of piperine in the physiological derangements of high fat diet induced obesity in Sprague Dawley rats. Chem Biol Interact. 2014;221:42-51.

12. Lam DD, Heisler LK. Serotonin and energy balance: molecular mechanisms and implications for type 2 diabetes. Expert Rev Mol Med. 2007;9:1-24.

13. Park U, Jeong H, Jo E, Park T, Yoon SK, Kim E, Jeong J, Um S. Piperine, a Component of Black Pepper, Inhibits Adipogenesis by Antagonizing PPARY Activity in 3T3-L1 Cells. J Agric Food Chem. 2012;60:3853-60.

14. Shah S, Dr. Shah G, Dr. Patel M, Dr. Singh S. Effect of Piperine in Obesity induced insulin resistance and type-Il diabetes mellitus in rats. Journal of Natural Remedies. 2010;10:116-22. 
15. Zhao Y, Liu J, Hao W, He Z, Zhu H, Ning Liangb, Ma KY, He W, Yang Y, Chen Z. Plasma cholesterollowering activity of piperine is mediated by inhibition on cholesterol absorption via down-regulation of intestinal ACAT2 and MTP. Journal of Functional Foods. 2018;49:465-71.

16. Bastaki M, Aubanel M, Bauter M, Cachet T, Demyttenaere J, Diop MM, Harman CL, Hayashi S, Krammer G, Li X, Llewellyn C, Mendes O, Renskers KJ, Schnabel J, Smith BPC, Taylor SV. Absence of adverse effects following administration of piperine in the diet of Sprague-Dawley rats for 90 days. FOOD AND CHEMICAL TOXICOLOGY. 2018;120:213-21.

17. Reagan Shaw $S$, Nihal $M$, Ahmad N. Dose translation from animal to human studies revisited. FASEB J. 2008;22:659-61.

18. Sun J, Ren F, Xiong L, Zhao L, Guo H. Bovine lactoferrin suppresses high-fat diet induced obesity and modulates gut microbiota in C57BL/6J mice. Journal of Functional Foods. 2016;22:189-200.

19. Zong X, Cao X, Wang H, Xiao X, Wang Y, Lu Z. Cathelicidin-WA Facilitated Intestinal Fatty Acid Absorption Through Enhancing PPAR-y Dependent Barrier Function. Front Immunol. 2019;10:1-12.

20. Zakłos-Szyda M, Pawlik N, Polka D, Nowak A, Koziołkiewicz M, Edek AP. Viburnum opulus Fruit Phenolic Compounds as Cytoprotective Agents Able to Decrease Free Fatty Acids and Glucose Uptake by Caco-2 Cells. Antioxidants. 2019;8:1-25.

21. Bischoff SC, Barbara G, Buurman W, Ockhuizen T, Schulzke JD, Serino M, Tilg H, Watson A, Wells JM. Intestinal permeability-a new target for disease prevention and therapy. BMC Gastroenterol. 2014;14:189.

22. Jwa H, Choi Y, Park U, Um S, Yoon SK, Park T. Piperine, an LXRa antagonist, protects against hepatic steatosis and improves insulin signaling in mice fed a high-fat diet. Biochem Pharmacol. 2012;84:1501-10.

23. Cifarell V, Abumrad NA. Intestinal CD36 and Other Key Proteins of Lipid Utilization: Role in Absorption and Gut Homeostasis. Comprehensive Physiology. 2018;8:493-507.

24. Wang TY, Liu M, Portincasa P, Wang DQH. New insights into the molecular mechanism of intestinal fatty acid absorption. Eur J Clin Invest. 2013;43:1203-23.

25. Drover VA, Nguyen DV, Bastie CC, Darlington YF, Abumrad NA, Pessin JE, London E, Sahoo D, Phillips MC. CD36 Mediates Both Cellular Uptake of Very Long Chain Fatty Acids and Their Intestinal Absorption in Mice. J Biol Chem. 2008;283:13108-15.

26. Hsu K, Storch J. Fatty Acid Transfer from Liver and Intestinal Fatty Acid-binding Proteins to Membranes Occurs by Different Mechanisms. The Journal of Biological Chemistry. 1996;271:13317-23.

27. Ockner RK, Manning JA. Fatty Acid-Binding Protein in Small-Intestine - Identification, Isolation, and Evidence for its Role in Cellular Fatty-Acid Transport. Journal of Clinical Investigation. 1974;54:32638.

28. Zhang L, Wang F, Wang J, Wang Y, Fang Y. Intestinal fatty acid-binding protein mediates atherosclerotic progress through increasing intestinal inflammation and permeability. J Cell Mol Med. 2020;24:5205-12. 
29. Stahl A. A current review of fatty acid transport proteins (SLC27). Pflugers Archiv European Journal of Physiology. 2004;447:722-7.

30. Shim J, Moulson CL, Newberry EP, Lin M, Xie Y, Kennedy SM, Miner JH, Davidson NO. Fatty acid transport protein 4 is dispensable for intestinal lipid absorption in mice. J Lipid Res. 2009;50:491500 .

31. Zhao Y, Liu J, Hao W, He Z, Zhu H, Liang N, Ma KY, He W, Yang Y, Chen Z. Plasma cholesterol-lowering activity of piperine is mediated by inhibition on cholesterol absorption via down-regulation of intestinal ACAT2 and MTP. Journal of Functional Foods. 2018;49:465-71.

32. DB M. S S, KR M: Role of Piperine as an Effective Bioenhancer in Drug Absorption. Pharmaceutica Analytica Acta 2018;09.

33. Hamilton MK, Boudry G, Lemay DG, Raybould HE. Changes in intestinal barrier function and gut microbiota in high-fat diet-fed rats are dynamic and region dependent. American Journal of Physiology-Gastrointestinal Liver Physiology. 2015;308:G840-51.

34. Guo G, Shi F, Zhu J, Shao Y, Gong W, Zhou G, Wu H, She J, Shi W. Piperine, a functional food alkaloid, exhibits inhibitory potential against TNBS-induced colitis via the inhibition of I kappa B-alpha/NFkappa B and induces tight junction protein (claudin-1, occludin, and ZO-1) signaling pathway in experimental mice. Human \& Experimental Toxicology; 2019.

35. Capaldo CT, Nusrat A. Cytokine regulation of tight junctions. Biochimica Et Biophysica ActaBiomembranes. 2009;1788:864-71.

36. Honggang Wang J, Dong PSJ, Liu LZYL, Gong LGJZ, Zhang WZW, Zhu NLAJ. Anti-mouse CD52 monoclonal antibody ameliorates intestinal epithelial barrier function in interleukin-10 knockout mice with spontaneous chronic colitis. Immunology. 2015;144:254-62.

37. Ma TY, Iwamoto GK, Hoa NT, Akotia V, Pedram A, Boivin MA, Said HM. TNF-a-induced increase in intestinal epithelial tight junction permeability requires NF-KB activation. American Journal of Physiology-Gastrointestinal Liver Physiology. 2004;286:G367-76.

38. Ma TY, Boivin MA, Ye D, Pedram A, Said HM. Mechanism of TNF-a modulation of Caco-2 intestinal epithelial tight junction barrier: role of myosin light-chain kinase protein expression. American Journal of Physiology-Gastrointestinal Liver Physiology. 2005;288:G422-30.

39. Guo G, Shi F, Zhu J, Shao Y, Gong W, Zhou G, Wu H, She J, Shi W. Piperine, a functional food alkaloid, exhibits inhibitory potential against TNBS-induced colitis via the inhibition of I kappa B-alpha/NFkappa B and induces tight junction protein (claudin-1, occludin, and ZO-1) signaling pathway in experimental mice. Human Experimental Toxicology. 2020;39:477-91.

40. Roig-Pérez S, Guardiola F, Moretó M, Ferrer R. Lipid peroxidation induced by DHA enrichment modifies paracellular permeability in Caco-2 cells. J Lipid Res. 2004;45:1418-28.

41. Hussain MM. Intestinal lipid absorption and lipoprotein formation. Curr Opin Lipidol. 2014;25:200-6.

42. Turner JR. Intestinal mucosal barrier function in health and disease. Nat Rev Immunol. 2009;9:799809. 


\section{Tables}

Due to technical limitations, table 1 is only available as a download in the Supplemental Files section.

Figures

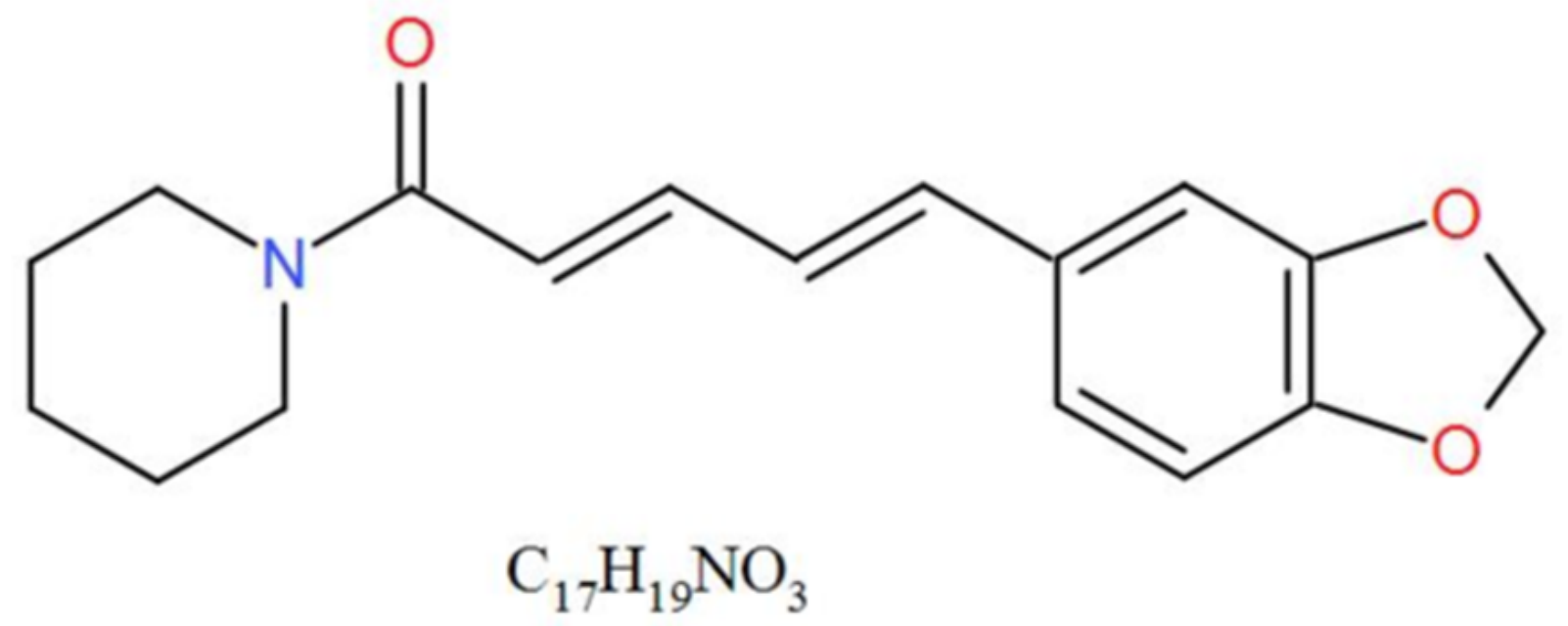

Figure 1

Structural formula of piperine 

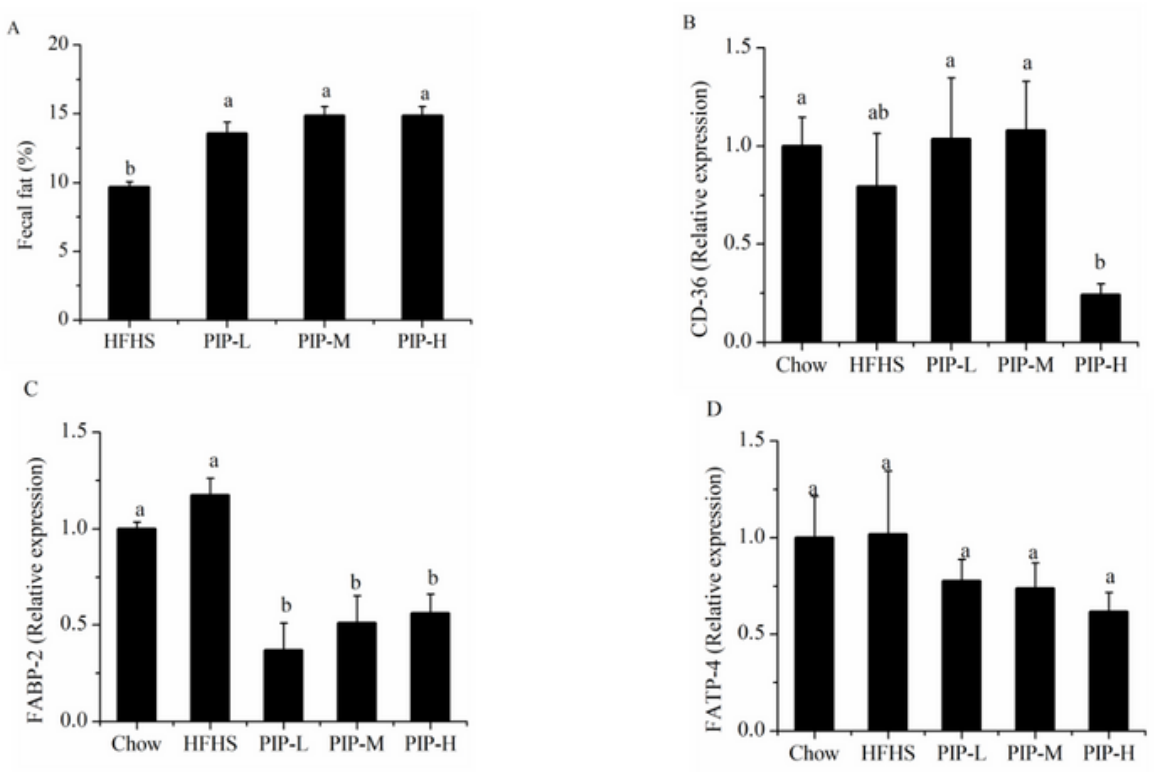

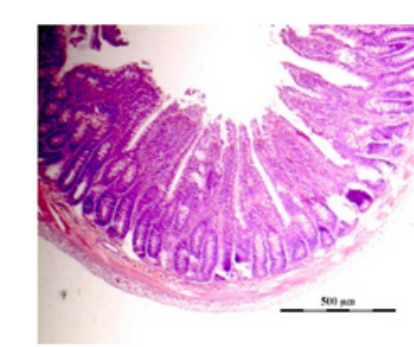

Chow

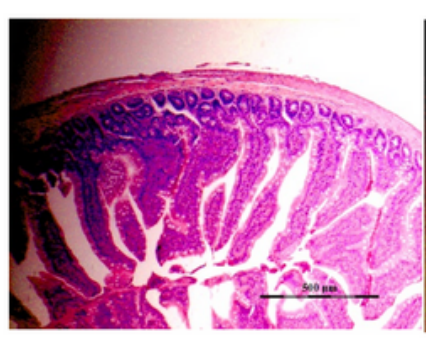

PIP-M
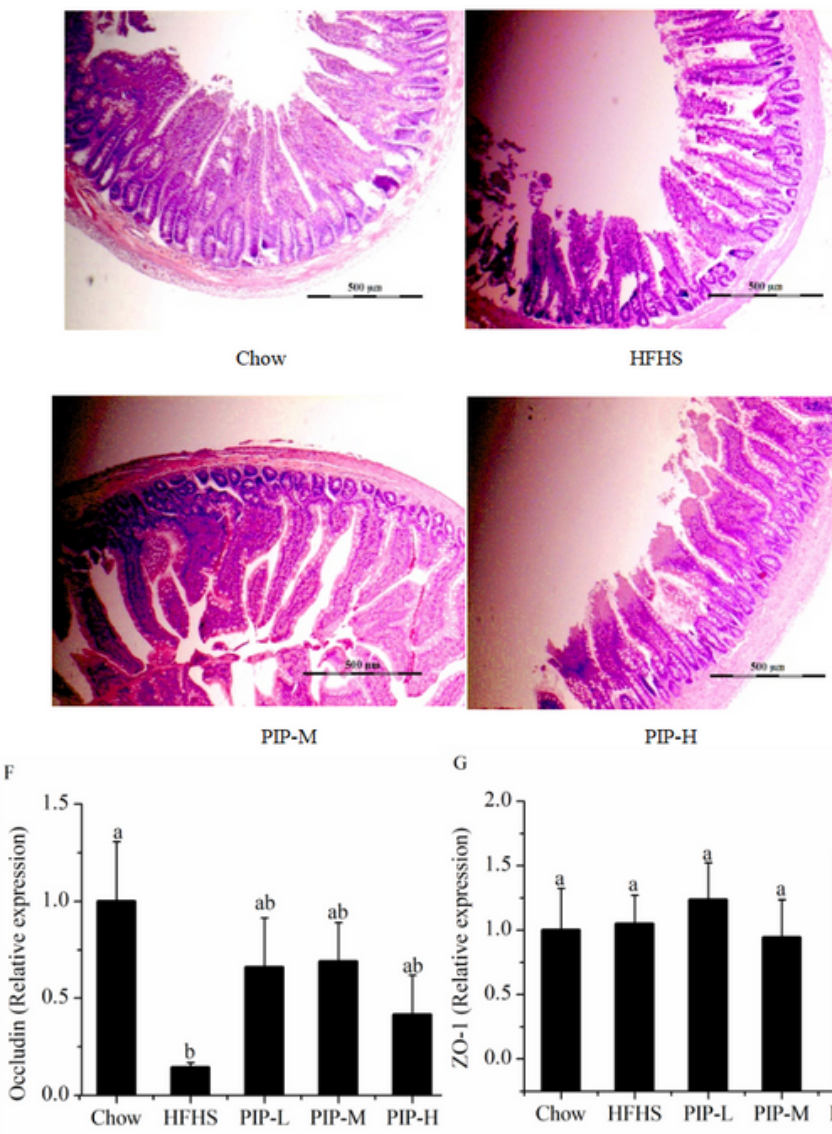

HFHS

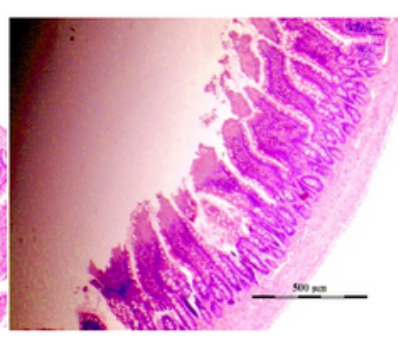

PIP-H
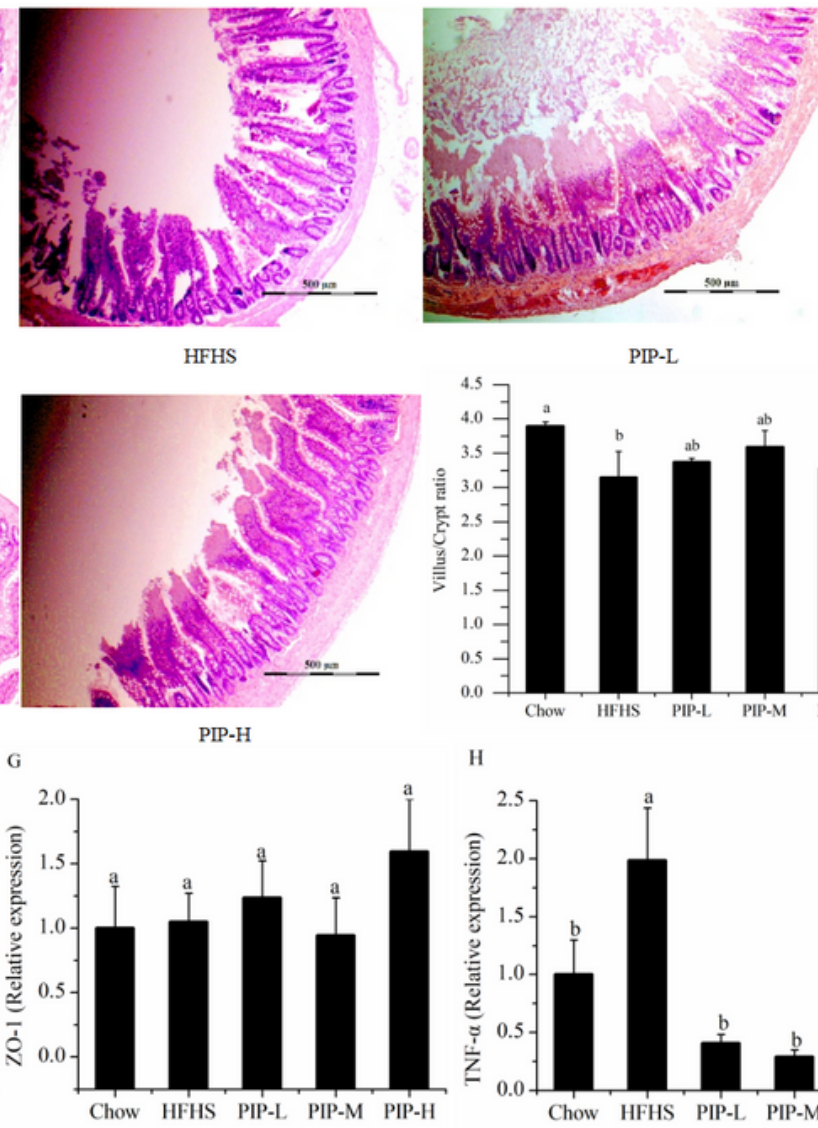

PIP-L

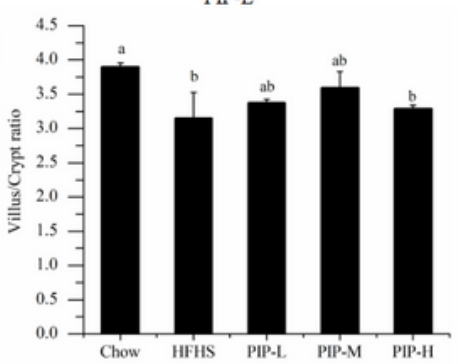

$\mathrm{H}$

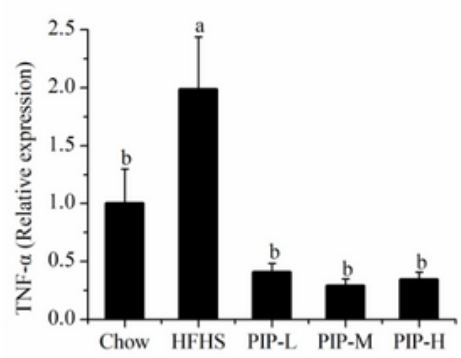

\section{Figure 2}

Influence of piperine on the jejunum of obesity rats. (A) Fatty acid content in feces of rats treated with HFHS and piperine under the same dietary conditions, $n=6$ rats. (B-D) qPCR performed to quantify fatty acid absorption related to mRNA levels. Results are presented relative to those of the Chow group, $n=9$ rats. (E) Representative H\&E-stained jejunal sections (magnification, 50x). Villus heights and crypt depths of the jejunum (values represent the average length of 30 structures (villi or crypt) per mouse, $n=3$ rats). 
(F-H) qPCR quantified occludin (F), ZO-1 (G), and TNF-a (H) mRNA abundance in the jejunum. Results are presented relative to those of the Chow group, $n=9$ rats. Data are expressed as mean \pm standard error of the mean (SEM). Values without a common letter are significantly different at $p<0.05$. HFHS, high-fat high-sugar; H\&E, hematoxylin-eosin; qPCR, quantitative polymerase chain reaction; ZO-1, Zonula occludens-1; TNF-a, tumor necrosis factor- $a$.
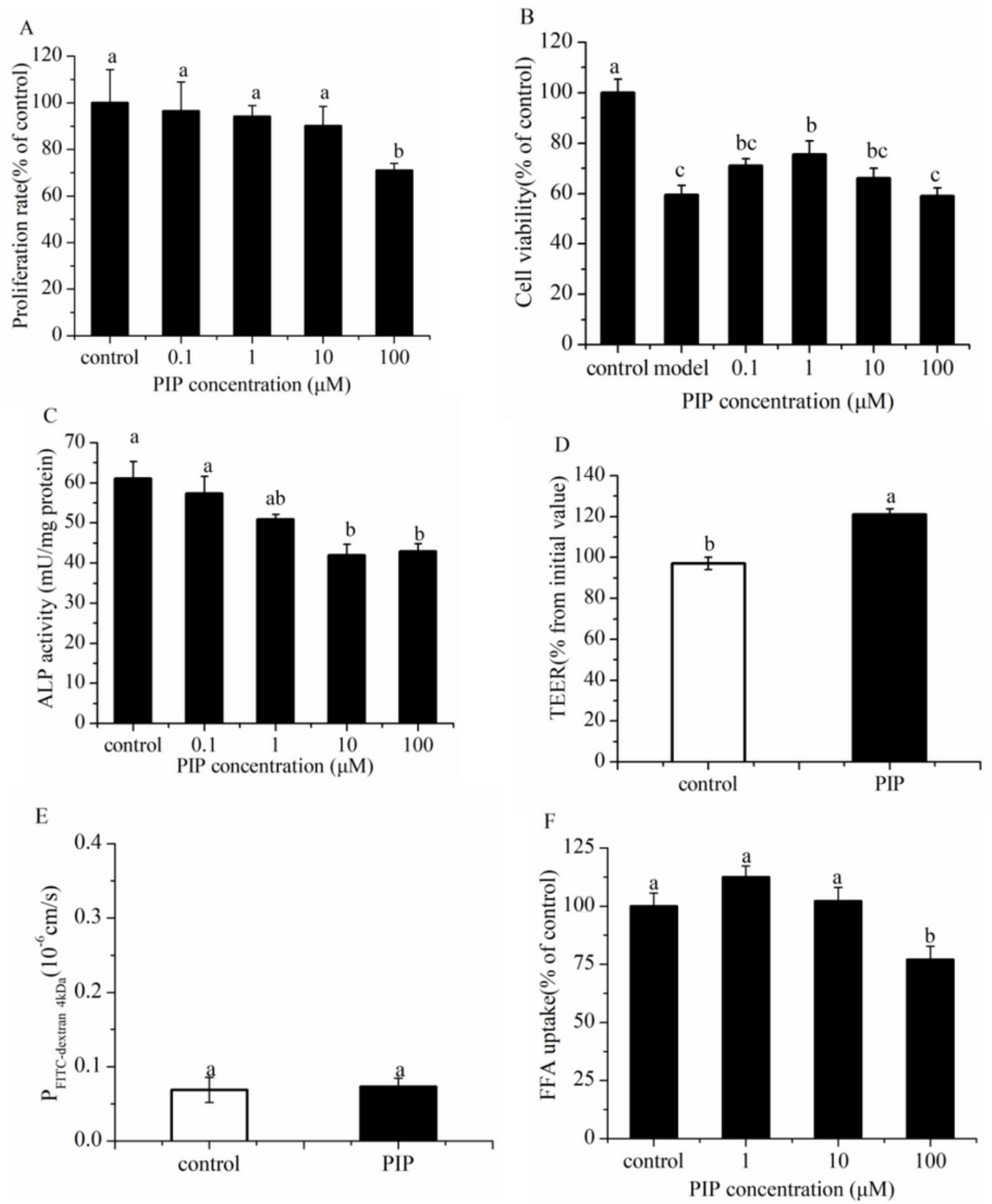

Figure 3 
Effect of piperine on proliferation, differentiation, and monolayer barrier function of Caco-2 cells. (A) Effect of piperine on the proliferation rate of Caco-2 cells, $n=5$. (B) Effect of piperine on the proliferation rate of LPS injured Caco-2 cells, $n=5$. (C) Effects of piperine on the ALP activity of Caco-2 cells, $n=5$. (D) Effect of piperine on TEER of a Caco-2 cell monolayer, $n=3$. (E) Effect of piperine on cellular permeability to FITC-dextran(4000Da), n=3. (F) Effect of piperine on fatty acid uptake by Caco-2 cells, n=3. Data are expressed as mean \pm standard error of the mean (SEM). Values without a common letter are significantly different at $p<0.05$. LPS, lipopolysaccharide; ALP, alkaline phosphatase; TEER, transepithelial electrical resistance.

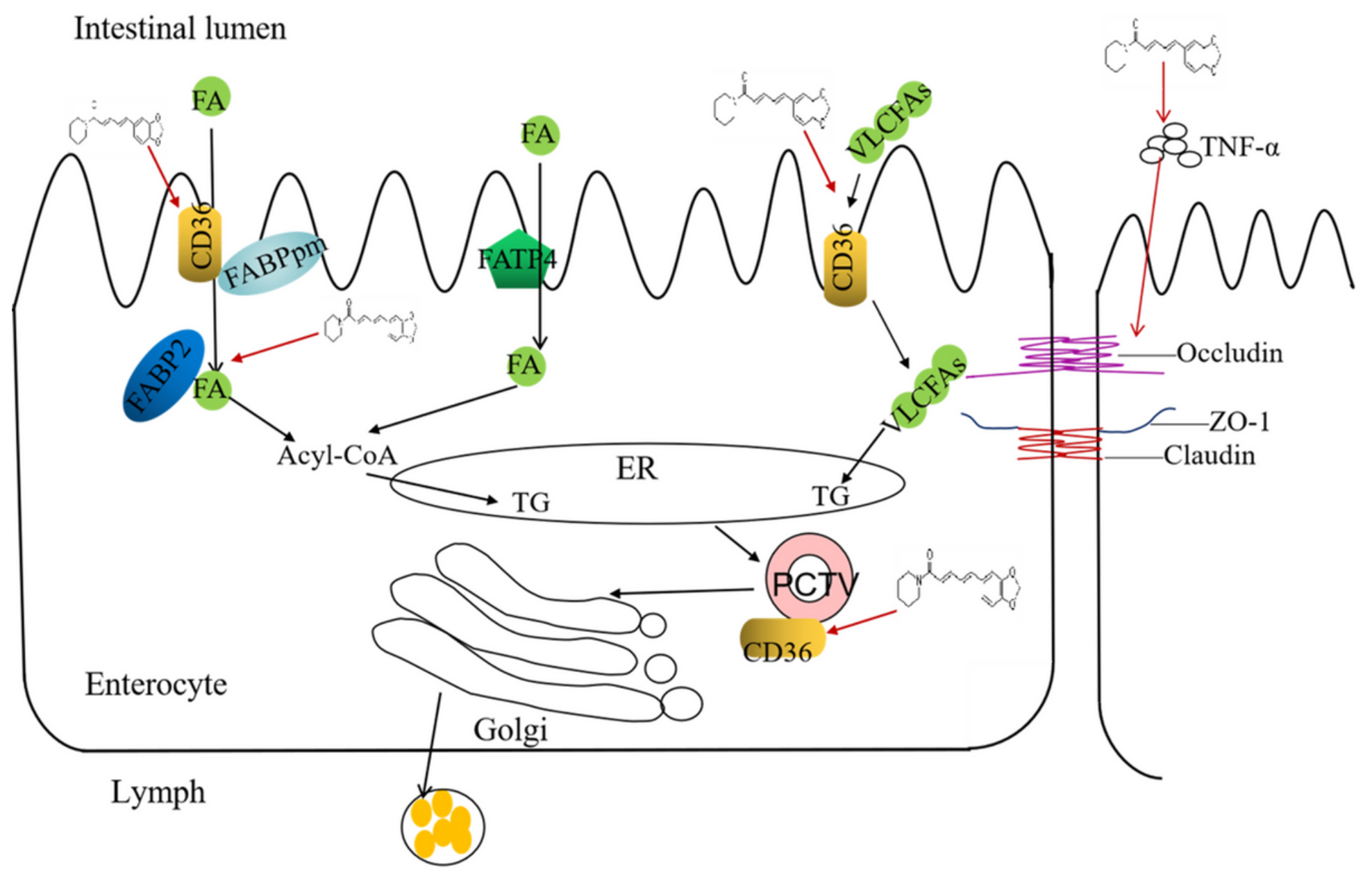

Figure 4

Effect of piperine on intestinal barrier function and absorption of fatty acids. Red arrows represent the process that may be affected by piperine. The black arrow represents the process of fatty acid absorption by intestinal epithelial cells. CD36 plays an important role in the absorption of VLCFAs[23]. CD36 alone or together with FABP pm accepts FA at the cell surface and then transports FA across the apical membrane of the enterocyte[24]. FA can also be transported into enterocytes by FATP4. Once at the inner side of the 
membrane, these FAs are bound by FABP2 before entering the metabolic pathways[24]. CD36 participates in the transport of prechylomicron transport vesicles (PCTVs) from the endoplasmic reticulum (ER) to the Golgi apparatus[23]. The chylomicron matures in the Golgi apparatus and is released across the basolateral membrane of the enterocyte to be transported into the lymph[41]. Combined with the results of this study, piperine downregulates the expression of CD36 and FABP2, and the process that piperine may affect is marked in the figure. Further studies are warranted to determine the detailed mechanism of action. Moreover, piperine downregulates the overexpression of TNF- $a$ and reduced TNF-a induced damage to occludin [35,42]. VLCFAs, very-long-chain fatty acids; FABP2, fatty acid-binding protein 2; FABP pm, plasma membrane-associated FABPs; FA, fatty acid; CD36, Cluster of differentiation 36; TNF-a, tumor necrosis factor-a.

\section{Supplementary Files}

This is a list of supplementary files associated with this preprint. Click to download.

- Table1.docx 